Journal of Physical Science, Vol. 29(1), 1-16, 2018

\title{
Assessment of Jatropha Oil as Insulating Fluid for Power Transformers
}

\author{
Abdelghaffar Amoka Abdelmalik, ${ }^{*}$ Paul Adeoluwa Abolaji and Hamzah Abubakar Sadiq \\ Department of Physics, Ahmadu Bello University, Zaria 810002, Nigeria \\ *Corresponding author: aaabdelmalik@abu.edu.ng
}

Published online: 25 April 2018

To cite this article: Abdelmalik, A. A., Abolaji, P. A. \& Sadiq, H. A. (2018). Assessment of Jatropha oil as insulating fluid for power transformers. J. Phys. Sci., 29(1), 1-16, https://doi.org/10.21315/jps2018.29.1.1

To link to this article: https://doi.org/10.21315/jps2018.29.1.1

\begin{abstract}
Seed-based oil has been identified as a viable alternative insulating fluid and a number of vegetable oils has been studied for that purpose. Among the seed-based oils studied is Jatropha oil which was reported to have suitable breakdown strength for high voltage application. However, most of the reports did not study the ageing characteristics of the oil and its compatibility with cellulose insulation. In this present study, the properties and ageing behaviour of a freshly prepared Jatropha oil and its compatibility with cellulose insulation paper were evaluated. The oil was purified through modified Dijkstra and Opstal purification method. The thermo-physical and dielectric studies of the oil was performed and thermal ageing of Kraft paper in the prepared ester fluid was performed. Thermally accelerated ageing was performed with an open beaker containing ageing catalysts, thermally upgraded insulation paper, and the fluid at $140^{\circ} \mathrm{C}$ for $120,240,360$ and $480 \mathrm{~h}$. Jatropha oil is a high temperature fluid with high specific heat capacity of $2.59 \mathrm{~kJ} \mathrm{~kg}^{-1} \mathrm{~K}$ and low dielectric loss of 0.009. The oil became polymerised with ageing in open cup. This may be due to thermo-oxidative degradation of the oil that progressed with ageing time. This is an indication that the oil is not suitable for use in free breathing power equipment.
\end{abstract}

Keywords: Dielectric fluid, insulating materials, paper insulation, dielectric loss, thermal ageing

\section{INTRODUCTION}

The idea of using natural ester fluid for electrical insulation in oil-filled electric equipment was conceived as far back as 1892. The development suffered a setback because the thermo-oxidative stability of the oil was inferior compared with conventional mineral oil-based transformer fluid. ${ }^{1}$ Mineral-based dielectric liquid 
is commonly used in power transformers as they have good insulating and cooling properties. However, they are known to be poorly-biodegradable and toxic. ${ }^{2}$ In a quest for alternative insulating fluids to mineral oil, a number of other fluids has been considered. They could not serve as better alternatives either because they could not meet the health and safety conditions, and are also more costly. Further efforts on the quest for alternative insulating fluids in the 90s show that vegetable oil with its environmental, safety and health benefits can still serve as a viable alternative insulating fluid. ${ }^{2}$

Although recent reports show that natural ester insulating fluid performs very well as an insulant and coolant, its high viscosity has posed a design challenge and its poor thermo-oxidative property has limited its application. ${ }^{3-5}$ Seed-based oils have two opposing properties: pour point and oxidative stability. These properties are dependent on the content of unsaturated fatty acids in the oil. The thermal and oxidative instability of the oil increase as the fatty acids in oil progresses from saturation to mono-unsaturation and poly-unsaturation. The higher the unsaturated fatty acid content, the higher the oxidative instability of the oil and poor oxidative stability is not desirable in insulating fluids. ${ }^{4}$ Conversely, the melting and pour points of the oil decrease with increase in the percentage of unsaturated fatty acid content.

The unsaturated fatty acids generally have lower melting and pour points than the saturated fatty acids. The high pour point of saturated fatty acid results from its uniform molecular shape, which enables the molecules to pack efficiently as it solidifies. Crystal formation is difficult in unsaturated fatty acids because of the bends and kinks introduced by the carbon-carbon double bonds. ${ }^{6}$ This limits the ability of the fatty acids to be closely packed. As a result, the more the unsaturation, the harder it becomes for the molecules to crystallise. This will have the consequence of lowering the pour point of the oil and low pour point is a desirable property of insulating fluids. ${ }^{7}$

Seed oil with higher percentage of unsaturated fatty acids has lower melting and pour points but poor stability to thermo-oxidation, while the seed oils with higher percentage of saturated fatty acids have better oxidative stability but higher melting and pour points. A number of vegetable oils has been studied for electrical insulation and there are commercially available natural ester dielectric fluids in the market such as Environtemp FR3 fluid produced from sunflower oil and Midel eN fluid produced from rapeseed oil., ${ }^{3,8-11}$ Most of the popular works on Jatropha oil were on its performance as biodiesel but there were also few works on its suitability as electrical insulating fluid. The oil was reported to possess properties that made it suitable for electrical insulation. ${ }^{12}$ 
It was reported that refined Jatropha oil has breakdown voltage of $35.8 \mathrm{kV}$ in a spherical electrode with $2.5 \mathrm{~mm}$ gap. ${ }^{13}$ This is within the specified breakdown voltage for fluid for high voltage insulation. However, the ageing behaviour of the oil was not reported. A number of non-edible seed-based oil including Jatropha oil is known to be available in Nigeria and an earlier attempt was made to study the viability of developing insulating fluid from Nigerian palm kernel oil. ${ }^{14}$ These oils are easily biodegradable with chemical structure that makes possible its modification to improve the thermal properties and oxidative stability. This paper therefore presents a study on the dielectric and thermal study of Jatropha oil, its ageing behaviour and its compatibility with insulation paper.

\section{EXPERIMENTAL}

\subsection{Sample Preparation}

A modified Dijkstra and Opstal purification method was adopted for the purification of the sample to obtain the purified Jatropha oil (PJOF) sample. A $200 \mathrm{ml}$ solution of crude Jatropha oil (CJO) sample was heated in a $500 \mathrm{ml}$ conical flask to $70^{\circ} \mathrm{C}$ and 8 vol $\%$ of $64 \mathrm{vol} \%$ aqueous citric acid solution was added gently and mixed thoroughly with a magnetic stirrer for $15 \mathrm{~min} .{ }^{15}$ An amount of $4 \mathrm{ml}$ of $8 \mathrm{vol} \% \mathrm{NaOH}$ solution was gently added and the mixture stirred at 400 $\mathrm{rpm}$ for $15 \mathrm{~min}$. The mixture was then dried in vacuum oven at $85^{\circ} \mathrm{C}$ for $30 \mathrm{~min}$ to reduce the water content. An amount of $2 \mathrm{~g}$ of silica gel was added to the mixture at $70^{\circ} \mathrm{C}$ and agitated for $30 \mathrm{~min}$ at $300 \mathrm{rpm}$ to prevent it from settling out. Fuller's earth was then added and continuously stirred for $30 \mathrm{~min}$ at $8 \mathrm{wt} \%$. The sample was then filtered with filter paper in vacuum oven at $85^{\circ} \mathrm{C}$.

\subsection{Thermal Properties}

To determine the cloud and pour points, the oil sample was placed in cylindrical test tube placed in an ice/salt bath. It was inspected at interval temperature and the temperature at which a distinct cloudiness appeared at the bottom of the test tube was recorded as the cloud point of the sample. The sample was then allowed to cool below $0^{\circ} \mathrm{C}$. The sample was tilted on the clamp at interval of $2^{\circ} \mathrm{C}$. The lowest temperature at which there was a movement of the oil was recorded as the pour point. ${ }^{16}$

The specific heat capacity of the oil can be determined by heating a sample of the oil in a beaker in vacuum to a certain temperature. The sample was quickly transferred into a lagged copper calorimeter with a copper stirrer. It was stirred for 
5 min and the final temperature was measured. The specific heat capacity of the oil sample was calculated from the formula: ${ }^{17}$

$$
\frac{\left(m_{c} m_{c}\right)\left(\theta_{s}-\theta_{1}\right)}{\left(m_{1}+m_{c}\right)\left(\theta_{2}-\theta_{3}\right)}
$$

where $m_{c}$ is mass of calorimeter, $m_{c}$ is mass of stirrer, $m_{1}$ is mass of calorimeter plus oil sample, $c_{c}$ is specific heat capacity of copper, $c_{s}$ is specific heat capacity of stirrer, $c_{0}$ is specific heat capacity of oil sample, $\theta_{1}$ is initial temperature of oil sample, $\theta_{2}$ is temperature of boiling oil sample, $\theta_{3}$ is final temperature of oil sample, calorimeter and stirrer.

\subsection{Rheology}

The density and specific gravity of oil samples were measured using specific gravity bottle at room temperature. The dynamic viscosity of a liquid is directly related to the heat transfer coefficient of the oil, and the viscosity is dependent on temperature change in the oil. The viscosity of the liquid samples was measured using Brookfield Viscometer with the appropriate spindle. The sample container was placed in water bath heated on a thermostatically controlled hot plate to vary the temperature of the sample within accuracy of $\pm 0.1^{\circ} \mathrm{C}$. Temperature sensor was immersed in the water bath to monitor the temperature. Each measurement was taken three times and the average calculated for accuracy.

\subsection{Dielectric Properties}

The conduction property of the liquid samples was studied using HM8118 LCR Bridge within frequency of 20 to $200 \mathrm{kHz}$. The liquid was placed in a parallel plate test cell. The dielectric constant and dissipation factor was measured from the bridge at ambient temperature of $30^{\circ} \mathrm{C}$. The study under frequency spectrum is to understand the loss mechanism that result in conduction in the liquid. The dissipation factor which is also referred to as loss tangent or tan $\delta$ is obtained from the ratio of the leakage current density to the capacitive current density.

\subsection{Compatibility with Insulation Paper Test}

Insulations in transformers are composite systems. They include oil, Kraft papers of different thicknesses, and pressboard of different thicknesses. The test for the compatibility of the solid insulation in the natural ester is very important. An ageing experiment was set up to test the ageing behaviour of Jatropha oil and its compatibility with insulating paper. A $250 \mathrm{ml}$ solution of Jatropha oil was first filtered using a medium fast filter paper with pore size of $125 \mathrm{~mm}$. The oil sample 
in a vessel was placed in vacuum oven and dried at a temperature of $70^{\circ} \mathrm{C}$ for $24 \mathrm{~h}$. The vacuum oven was then allowed to cool to ambient temperature for $24 \mathrm{~h}$ and the oil sample was transferred to the ageing vessel.

The thermally upgraded insulating paper with thickness $0.255 \mathrm{~mm}$ and $15 \mathrm{~g}$ weight was vacuum dried in vacuum oven at $85^{\circ} \mathrm{C}$ for $24 \mathrm{~h}$. The paper was then impregnated with Jatropha oil in vacuum for impregnation using the capillary method. The ageing vessel containing the oil sample, $15 \mathrm{~g}$ of $0.255 \mathrm{~mm}$ thick Kraft paper, galvanised steel strip $(3.5 \mathrm{~cm} \times 3.5 \mathrm{~cm})$, copper strip $(3.5 \mathrm{~cm} \times 4.5 \mathrm{~cm})$, aluminium strip $(4 \mathrm{~cm} \times 4 \mathrm{~cm})$, and mild steel was placed in vacuum oven at room temperature. The galvanised steel represents the core of a transformer, while the copper and aluminium strip represents the conducting coils. ${ }^{17}$ The Kraft insulation paper samples were packed together and placed in between the metals sheets. The vessel was thermally aged at $150^{\circ} \mathrm{C}$ for $480 \mathrm{~h}$. Samples of aged cellulose papers were taken out of the vessel for analysis.

\section{RESULTS AND DISCUSSIONS}

Figure 1 shows the Jatropha oil samples under study. Table 1 shows the measured values of the density, dynamic viscosity, cloud point, pour point, flash point and specific heat capacity of the CJO sample, PJOF and compared with that of mineral oil.

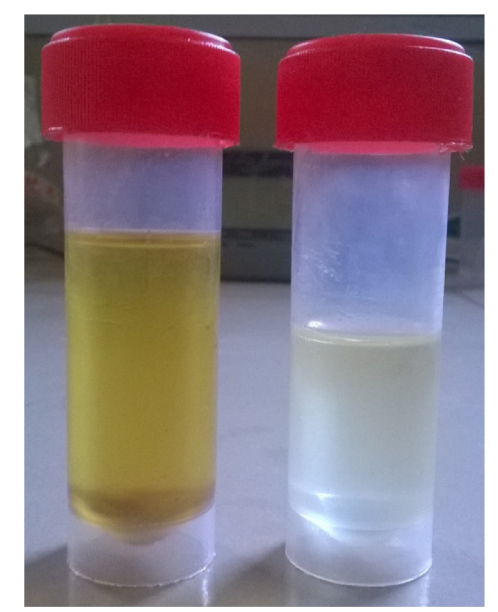

Figure 1: Samples of CJO (left) and PJOF (right).

PJOF was found to have a density and dynamic viscosity of $890 \mathrm{~kg} \mathrm{~m}^{-3}$ and $35.6 \mathrm{mPas}$ respectively at $30^{\circ} \mathrm{C}$. The viscosity is higher compared with mineral oil but comparable to that of ester-based insulating fluids. The pour point of Jatropha 
oil was determined to be $-6^{\circ} \mathrm{C}$. It can be further improved with the addition of pour point depressant. The flash point of the oil sample was determined to be $195^{\circ} \mathrm{C}$; the flash point is higher than the minimum specification for mineral oil based insulating fluid..$^{18}$ The specific heat capacity of the oil was determined to be $2.59 \mathrm{~kJ} \mathrm{~kg}^{-1} \mathrm{~K}$ which is much higher compared with mineral oil with specific heat capacity of $1.67 \mathrm{~kJ} \mathrm{~kg}^{-1} \mathrm{~K}$.

Table 1: Experimental results of Jatropha oil in comparison with mineral oil.

\begin{tabular}{lccc}
\hline Properties & CJO & PJOF & Mineral oil \\
\hline Density at $30^{\circ} \mathrm{C}\left(\mathrm{kg} \mathrm{m}^{-3}\right)$ & 891 & 890 & 880 \\
Dynamic viscosity at $30^{\circ} \mathrm{C}(\mathrm{mPa} . \mathrm{s})$ & 38.5 & 35.6 & 12.3 \\
Cloud point $\left({ }^{\circ} \mathrm{C}\right)$ & 6 & 7 & -10 \\
Pour point $\left({ }^{\circ} \mathrm{C}\right)$ & -5 & -5 & -34 \\
Flash point $\left({ }^{\circ} \mathrm{C}\right)$ & 196 & 195 & 149 \\
Specific heat capacity $\left(\mathrm{kJ} \mathrm{kg}^{-1} \mathrm{~K}\right)$ & 2.54 & 2.59 & 1.67 \\
\hline
\end{tabular}

Dielectric constants of the oil samples were obtained from the measured capacitance with programmable LCR Bridge. The dielectric constant of the samples was plotted against frequency as shown in Figure 2. The response of dielectric constant of crude and purified samples to frequency change have similar pattern. The dielectric constant of $\mathrm{CJO}$ at $100 \mathrm{~Hz}$ is 3.7 while that of PJOF is 3.8 at the same frequency as shown in Table 2.

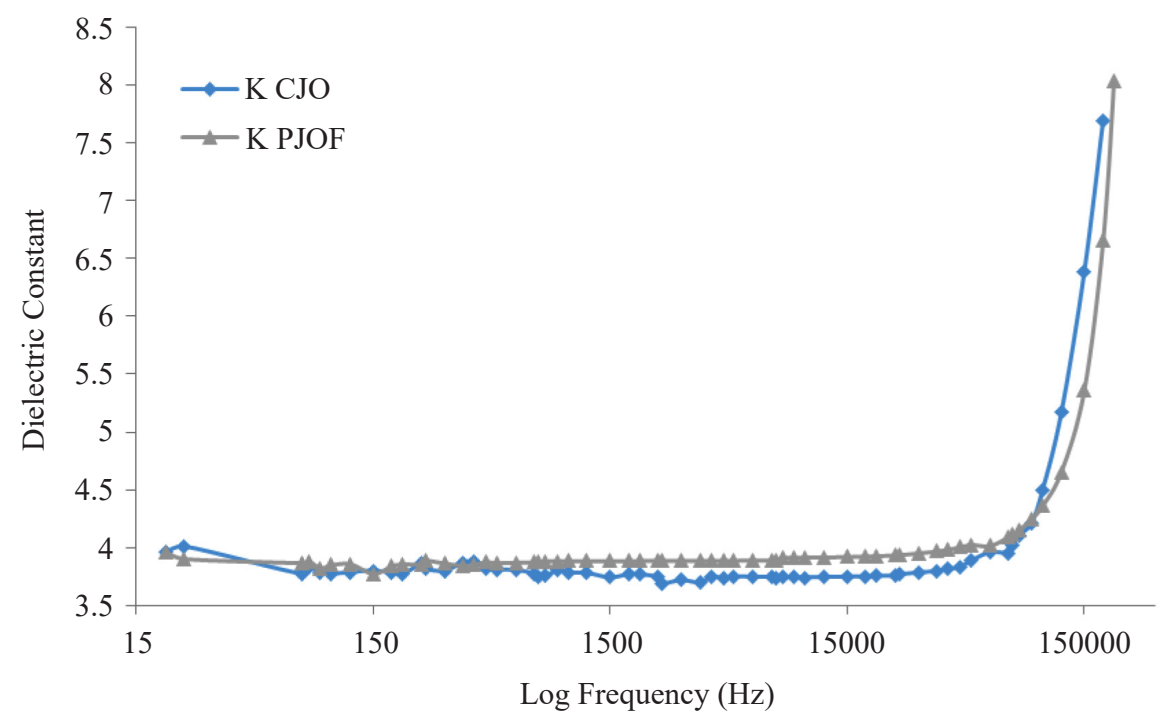

Figure 2: Variation of the dielectric constant of Jatropha oil with logarithm of frequency. 


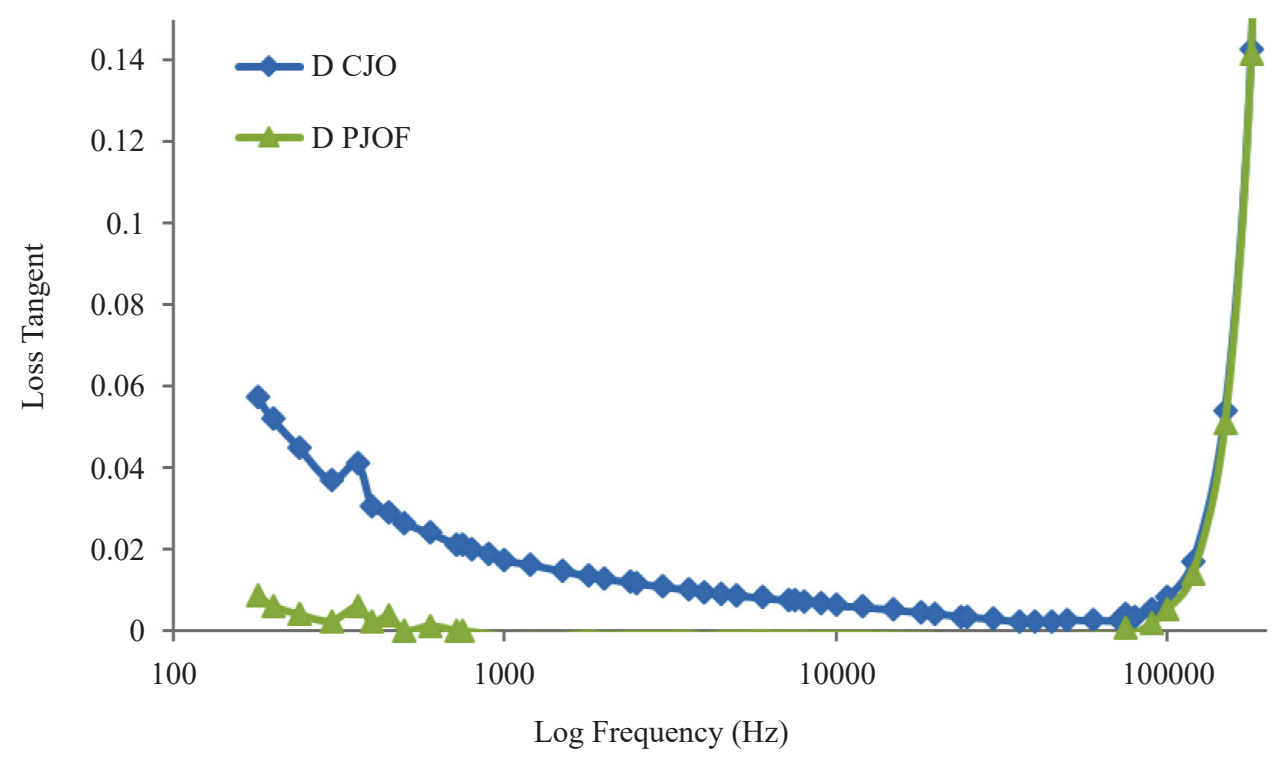

Figure 3: Variation of the loss tangent of Jatropha oil with logarithm of frequency.

The semi-log plot of loss tangent measure with the LCR bridge as a function of frequency for the oil samples is shown in Figure 3. The measurement was within the frequency range of $20 \mathrm{~Hz}$ to $200 \mathrm{kHz}$. The measured tan $\delta$ (dielectric loss) for PJOF from $900 \mathrm{~Hz}$ to $60 \mathrm{kHz}$ was below detection limit of the measurement instrument. It was observed from the graph that $\tan \delta$ values of the sample reduced after purification at low frequencies. This suggests that purification has a significant influence on the loss factor of the oil sample. Tan $\delta$ for crude and refined oil were 0.08 and 0.009 respectively as shown in Table 2 . Refining process reduced the loss tangent by a factor of $10^{-1}$. This is an indication that the purification process with fuller's earth may have been adsorb some conducting impurities in the oil samples. This resulted in improved dielectric loss characteristics of the oil.

The AC conductivity, which is sometimes considered the effective electrical conductivity of a dielectric material since it includes all the loss factors in the dielectric, is a function of the measured dielectric constant and dissipation factor. An effective AC conductivity may be defined as the ratio of the leakage current density to the magnitude of the field at a given frequency arising from conduction and dipole orientation losses. The AC conductivity of the samples was evaluated using the equation: ${ }^{19}$

$$
\sigma=\frac{\bar{J}_{1}}{\bar{E}}=\omega \varepsilon^{\prime} \tan \delta
$$


where $\sigma$ is the electrical conductivity, $\varepsilon^{\prime}(\omega)$ is the real component of the relative permittivity of the liquid, $\tan \delta(\omega)$ is the loss tangent which measures the energy dissipated by the dielectric liquid, $\varepsilon^{\prime}(\omega)$ and $\tan \delta(\omega)$ are function of angular frequency, $\omega, \bar{J}_{t}$ is the loss current density, and $\bar{E}$ is the electric field. The electrical conductivity of PJOF as shown in Table 2 falls within insulating material category but is about 2 order of magnitude higher than mineral insulating oil.

Table 2: Measured parameters at $30^{\circ} \mathrm{C}$.

\begin{tabular}{lccc}
\hline Properties & CJO & PJOF & Mineral oil \\
\hline Dielectric constant & 3.7 & 3.8 & 2.1 \\
Loss tangent at $30^{\circ} \mathrm{C}$ & 0.08 & 0.009 & 0.0002 \\
$\mathrm{AC}$ conductivity $\left(\mathrm{S} \mathrm{m}^{-1}\right)$ & $2.98 \times 10^{-9}$ & $3.44 \times 10^{-10}$ & $1.40 \times 10^{-12}$ \\
\hline
\end{tabular}

The motion of the charged particles responsible for conduction in the liquid may have resulted from the dissociation of ionic and solid impurities in the liquid. Besides causing conduction current, they give rise to polarisation of the dielectric material. If the total loss in the liquid is considered as a combination of DC conductivity and loss due to polarisation, the measured dielectric loss can be express as:

$$
\sigma(\omega)=\sigma_{0}+\omega \varepsilon_{0} \varepsilon^{\prime \prime}
$$

where $\sigma_{0}$ is the DC conductivity, $\omega \varepsilon_{0} \varepsilon "$ is loss due to polarisation, and $\varepsilon^{\prime \prime}$ is the relative permittivity. Polarisation of charged particles in CJO and PJOF may be responsible for the decrease in the effective conductivity of the oil samples. The lower conductivity of the purified sample compared with the crude sample is an indication that the purification process was able to eliminate some of the conducting impurities.

The relative permittivity and $\tan \delta$ of the dried and degassed insulating paper, unaged oil-impregnated paper and aged oil-impregnated paper at various ageing time is plotted over a frequency range of $10^{1}-10^{5} \mathrm{~Hz}$ in Figures 4 and 5 . Impregnation of the dried paper with the freshly prepared ester sample shows an increase in the permittivity and $\tan \delta$ of the composite material. The increase in permittivity is expected as oil impregnation makes it possible for the composite material to store more charges. The increase in dielectric loss after oil impregnation may have resulted from electrical conduction through the oil that fill the pores between the fibres of the insulating paper. The oil provides a channel for the transport of ionic impurities in the composite material. 


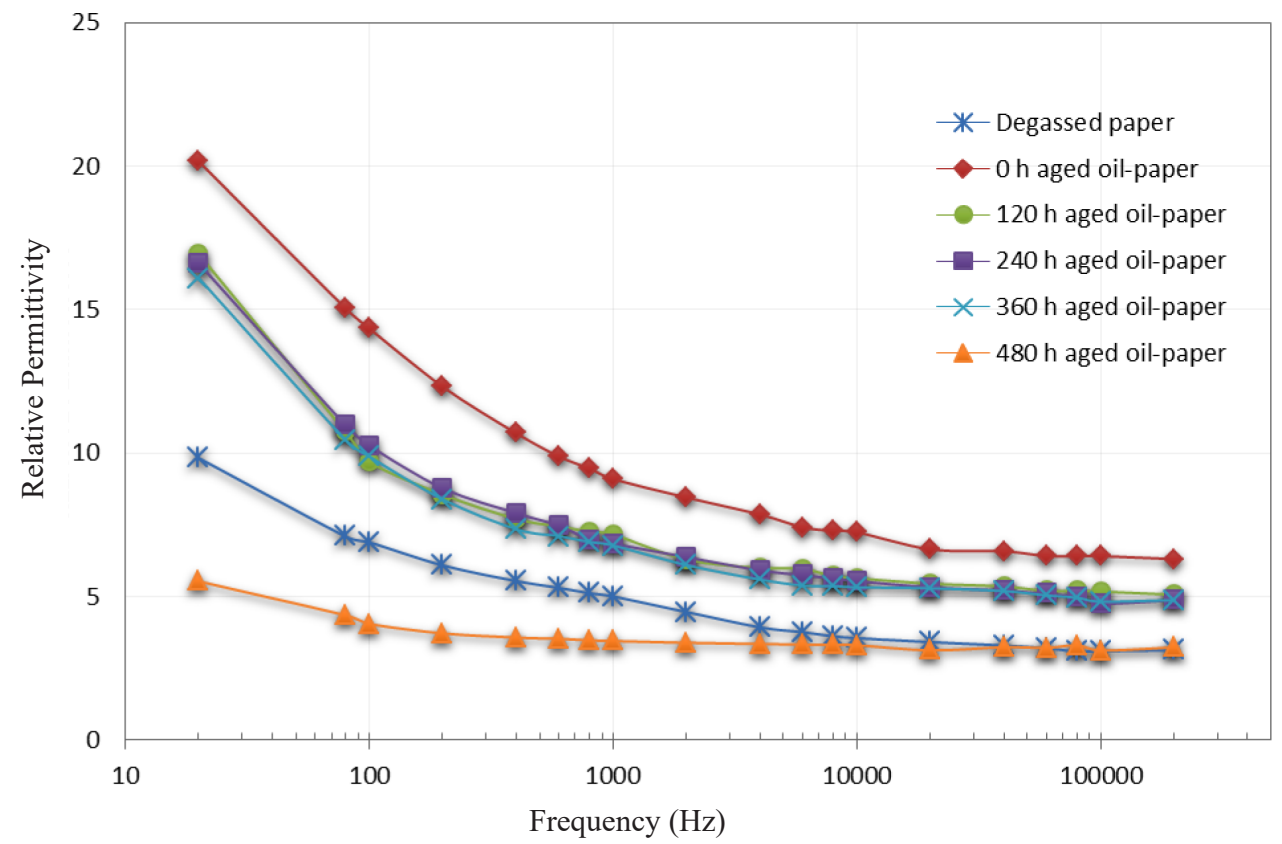

Figure 4: Graph of the permittivity for Jatropha oil.

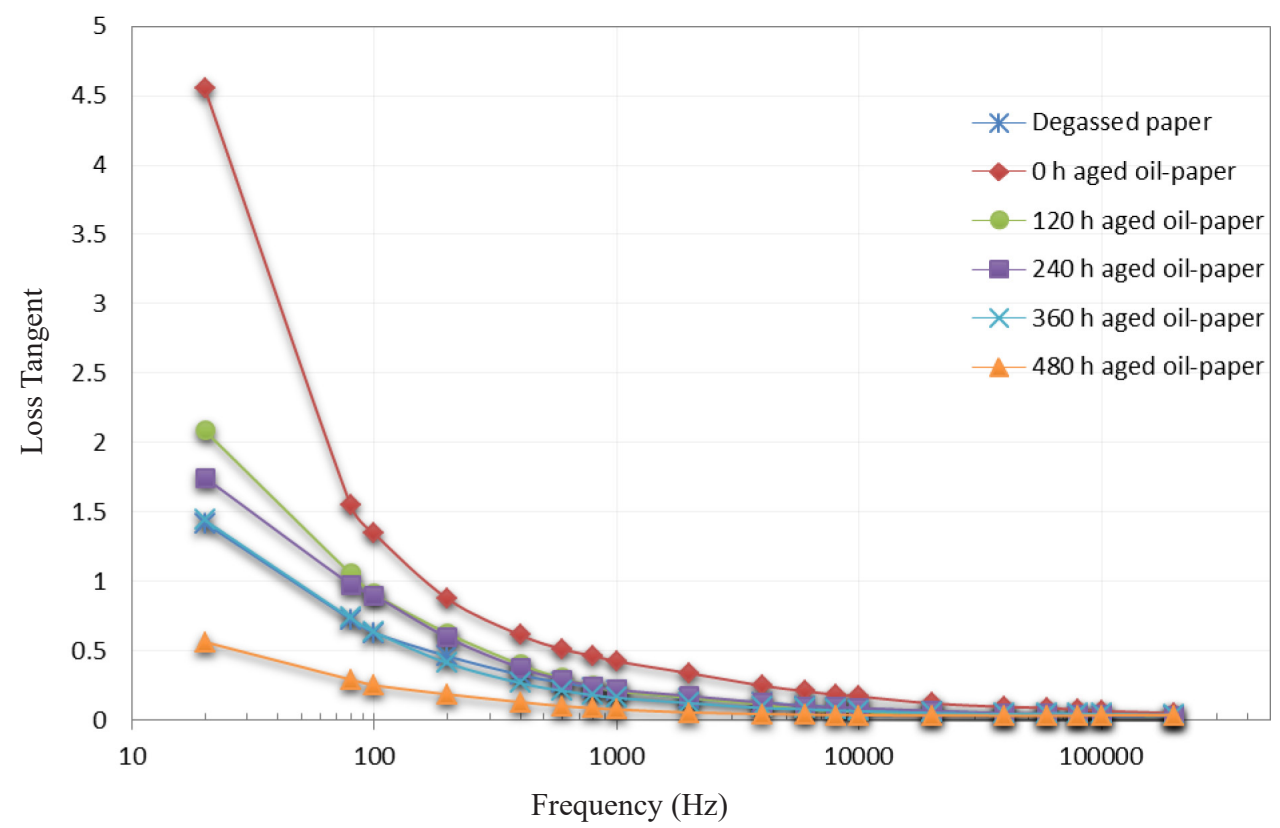

Figure 5: Graph of the loss tangent for Jatropha oil. 
Ageing for $120 \mathrm{~h}$ shows a decrease in magnitude of relative permittivity and $\tan \delta$ of the composite material. Ageing up till $360 \mathrm{~h}$ did not seem to have more impact on the magnitude of relative permittivity. However, further ageing for $480 \mathrm{~h}$ shows a significant decrease in relative permittivity. The values were lower than that of the dried paper. The decreasing $\tan \delta$ value with ageing is an indication that the dielectric loss of the sample decrease with ageing of the impregnated paper within the studied ageing time. This may have resulted from the polymerisation of the oil in the paper, making the composite system to behave more like a polymer than oil-impregnated paper.

The plot of real and imaginary (denoted with "imag" in figure) parts of the relative permittivity is shown in Figure 6. A close observation of the plot shows a logarithmic shift in both magnitude and frequency of the response towards low frequency with ageing.

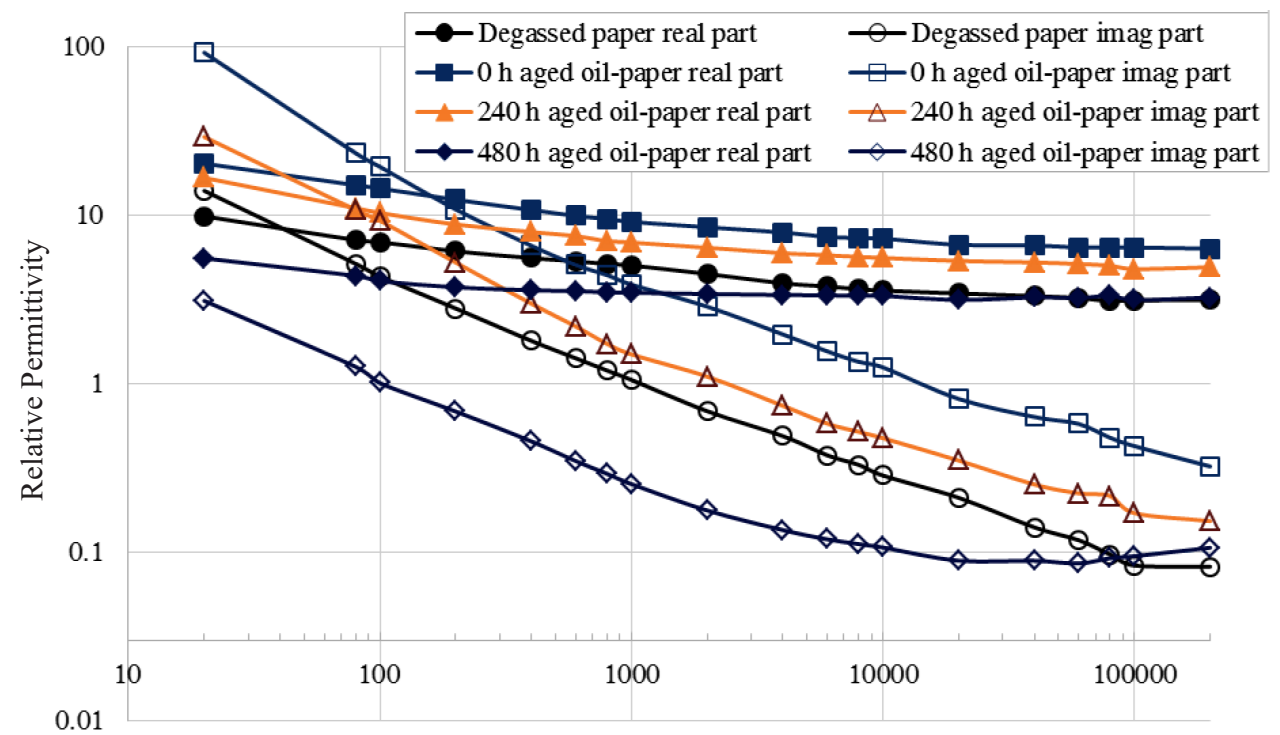

Frequency $(\mathrm{Hz})$

Figure 6: Real and imaginary relative permittivity of aged oil impregnated papers at $30^{\circ} \mathrm{C}$.

It is known that the weak polar nature of natural ester makes it possess strong affinity for water. ${ }^{20}$ This may have caused water to move from the Kraft paper insulation into the natural ester fluid in larger quantities. The generated water during pyrolysis process is absorbed by the ester. This cumulative equilibrium shift of water effectively dries the paper and reduces ageing due to thermo-hydrolytic degradation. The paper is protected from ageing through the water scavenging 
and steric hindrance of the cellulosic hydrolytic reaction by natural esters. ${ }^{21-23}$ The drying of the paper may have been responsible for the observed decrease in the dielectric constant and dielectric loss of the impregnated paper. The water is then consumed by hydrolysis of the natural ester, producing free fatty acids. This may react with the cellulose backbone via transesterification during thermal ageing thereby protecting the cellulose from hydrolysis. ${ }^{24}$

Oil-impregnated Kraft paper is an insulating paper whose voids between the paper fibres are filled with oil to improve the insulation strength. Ageing of esterimpregnated paper has been reported to be slower compared with mineral oilimpregnated paper. ${ }^{25}$ Ester impregnated paper behaves like a material with cavities filled with the impregnated oil and all its content. The cavities can also be referred to as clusters.

Two different contributions can be observed from the dielectric response of the oil-impregnated paper on the application of electric field to the composite material when placed between two electrodes as shown in Figure 6. There is a frequency independent real part of the permittivity at the high frequency end of the spectra. This response includes dielectric relaxations taking place in the void at frequencies higher than $10^{4} \mathrm{~Hz}$, together with atomic and electronic polarisations from quantum resonances. This is referred to as intra-cluster interaction as the dipoles interact within the voids. At lower frequencies, there is a dispersion in the relative permittivity. Within this region, there are inter-cluster interactions between the voids and the charges in one void are able to drift into another void.

The real and imaginary parts were observed to cross over where the real and imaginary parts of the relative permittivity have the same values. This is the frequency where the interactions of the negative and positive charge carriers in the composite material changed from intra-cluster to inter-cluster interactions and it will be referred to as the cross-over frequency in this work. Below the crossover frequency, the imaginary part has higher value compared with the real part and after the cross-over frequency, the reverse is the case. There appears to be links between the two processes before and after the cross-over frequency as earlier reported by Abdelmalik et al. ${ }^{26}$ Dissado-Hill cluster model was used to explain the interaction. ${ }^{27}$ Before the cross-over frequency, there is the intra-cluster interaction of the negative and positive charge carriers that are bound together as a dipole. Below the cross-over frequency, the negative and positive charge carriers become independent carriers that will result in quasi-dc (q-dc) process at much lower frequencies. This can also be called inter-cluster interaction. The cross-over frequency can be said to correspond to the onset time (inverse of cross over frequency) for the separation of ionic charges across the width of the oil 
filled cavity. This feature of the dielectric response of oil-paper insulation was observed to change with ageing. The cross-over frequency was observed to be shifting towards the low frequency end with ageing.

A plot of the onset of frequency of separation of ionic charges with ageing time is shown in Figure 7. The freshly impregnated oil-paper has a cross-over frequency of $150 \mathrm{~Hz}\left(1.11 \times 10^{-3} \mathrm{~s}\right)$ and after ageing of $120 \mathrm{~h}$, the frequency reduced to $90 \mathrm{~Hz}\left(1.77 \times 10^{-3} \mathrm{~s}\right)$. The frequency did not seem to have much variation for ageing from $120 \mathrm{~h}$ to $360 \mathrm{~h}$. After $480 \mathrm{~h}$ of ageing, the frequency reduced to $10 \mathrm{~Hz}$ $\left(1.59 \times 10^{-2} \mathrm{~s}\right)$.

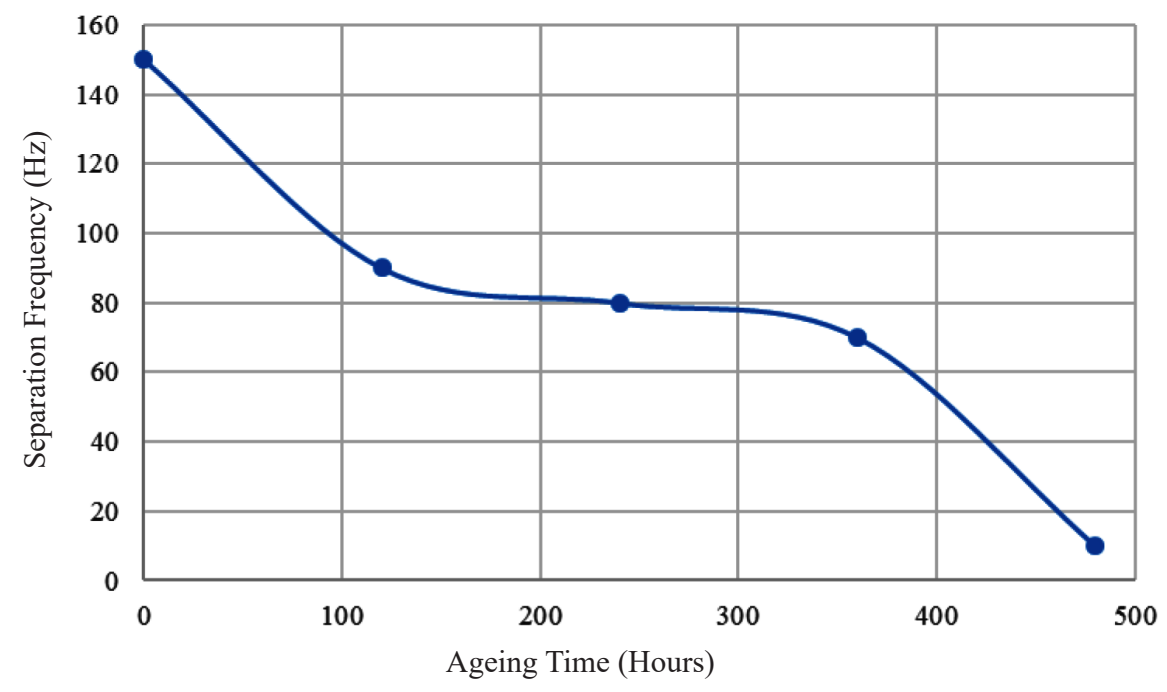

Figure 7: A plot of frequency of separation of ionic charges with aged time.

The plot shows that the separation frequency did not follow a simple relation. Dielectric response of the aged ester-paper is dependent on various factors which includes the ageing characteristic of both the oil and the paper. ${ }^{26}$ This makes the analysis of dielectric response of ester-paper system complex. It can be observed from the relationship in Figure 7 that the ageing behaviour of Jatropha oil-paper system may be difficult to predict from the cross over frequency. At $480 \mathrm{~h}$ of ageing for example, the oil sample was observed to have undergone significant polymerisation. The by-products of the process may have also contributed to the dielectric response of the oil-paper, thereby influencing the separation frequency. Ageing may have resulted in small modification of the morphology of the fibres of the paper leading to frequency shift of the dielectric response. A decrease in the magnitude at the separation frequency in the ester-paper system may be an indication of a decrease in the amount of ionic species contributing to the loss processes as the material aged with time. 


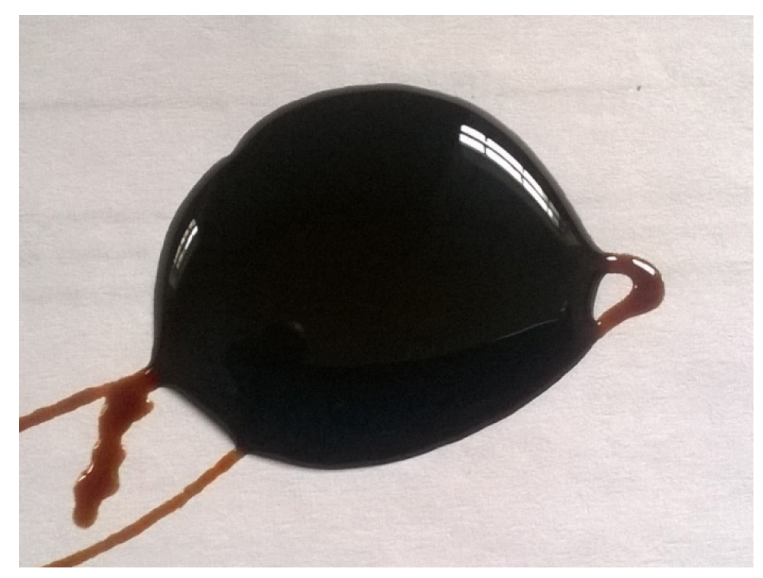

Figure 8: Polymerised aged sample after $480 \mathrm{~h}$.

The ageing took place in an oven that allowed free circulation of air. The oil was observed to have undergone changes with ageing. The colour turned dark and after $480 \mathrm{~h}$, the oil turned to a gel-like substance as shown in Figure 8. The FTIR spectra analysis of the aged oil samples reveal a peak within the fingerprint region. The spectra show that the aged samples exhibit a peak at $960 \mathrm{~cm}^{-1}$. The magnitude of the peak increased with ageing. This may have been due to thermal polymerisation of the oil. Jatropha oil is $86 \%$ unsaturated fatty acid. A search on FTIR database revealed that the peak corresponds to cyclic carboxylic functional group. ${ }^{28}$ The carbon-carbon double bond $(-\mathrm{C}=\mathrm{C}-)$ in the triglyceride has a strong sigma bond and a weak $\pi$ bond. At high temperature, the $\pi$ bond in the carbon-carbon double bond of the oil may have been modified in the presence of air and cause crosslinking of the fatty acid chains. They may have resulted in the formation of cyclic carboxyl group. The metal catalyst in the ageing setup may have also contributed to the acceleration of the thermal degradation of the oil. The increasing magnitude is an indication that the thermal polymerisation process progressed with time. This is an indication that even though the property of freshly prepared Jatropha oil suggests that it is suitable for high voltage insulation, it may only be useful in hermetically sealed system but not in free-breathing power equipment. Its use in a system open to air may require further modification to improve its oxidative stability.

\section{CONCLUSION}

The properties of freshly prepared Jatropha oil sample seem to be very interesting in agreement with earlier reports. Jatropha oil is a high temperature liquid when compared with mineral oil judging from its flash point. ${ }^{18}$ It has viscosity that is 
comparable to the market available natural ester dielectric fluids. They share similar dielectric constant, low loss and pour point less than $0^{\circ} \mathrm{C}$. It also has a low dielectric loss. Impregnated cellulose paper with the prepared ester fluid (PJOF fluid) shows a dielectric loss that decreased with ageing contrary to most report of oil-impregnated paper ageing with increase in dielectric loss. This is thought to be due the greater affinity of natural ester fluid for moisture, combined with hydrolysis, which caused water to move from the Kraft paper insulation into the fluid in larger quantities. This cumulative equilibrium shift of water effectively dries the paper, thereby reducing ageing rate due to thermo-hydrolytic degradation. However, the aged ester fluid at $140^{\circ} \mathrm{C}$ in air atmosphere was polymerised and the thermal polymerisation process progressed with ageing time $480 \mathrm{~h}$. This is an indication that the oil may only be useful in hermetically sealed system but not in free-breathing power equipment. Modifying the oil by converting the double bond site to an epoxy group which is more resistive to thermal degradation may improve the thermo-oxidative properties of the oil. Such modification can make it an allpurpose industrial fluid.

\section{REFERENCES}

1. McShane, C. P. (2002). Vegetable-oil-based dielectric coolant. IEEE Ind. Appl. Mag., 8(3), 34-41, https://doi.org/10.1109/2943.999611.

2. Fofana, I. et al. (2001). Retrofilling condition of HV transformers. IEEE Electr. Insul. Mag., 17, 17-30, https://doi.org/10.1109/57.917528.

3. Oommen, T. V. (2002). Vegetable oils for liquid-filled transformers. IEEE Elect. Insul. Mag., 18(1), 6-11, https://doi.org/10.1109/57.981322.

4. Bertrand, Y. \& Hoang, L. C. (2003). Vegetal oils as substitute for mineral oils. Paper presented at the Proceedings of the 7th International Conference on Properties and Applications of Dielectric Materials, 1-5 June, https://doi. org/10.1109/ICPADM.2003.1218460.

5. Tenbohlen, S. et al. (2008). Application of vegetable oil-based insulating fluids to hermetically sealed power transformers. Cigre session, paper A2102. 21, rue d'Artois, F-75008, Paris.

6. Gunstone, F. D. et al. (2007). Nonfood uses of oils and fats. In Gunstone, F. D. et al. (Eds). The lipid handbook, 3rd ed. New York: CRC Press, 591635.

7. Hwang, H. \& Erhan, S. Z. (2001). Modification of epoxidized soybean oil for lubricant formulations with improved oxidative stability and low pour point. J. Am. Oil Chem. Soc., 78, 1179-1184, https://doi.org/10.1007/s11745-0010410-0. 
8. Li, J. et al. (2007). Dielectric properties of rapeseed oil paper insulation. Ann. Rep. Conf. Elect. Insul. Dielectr. Phen., 500-503.

9. Li, X., Li, J. \& Sun, C. (2006). Properties of transgenic rapeseed oil based dielectric liquid. Paper presented at the Proceedings of the IEEE International Conference on Dielectric Liquids.

10. Suleiman, A. A. et al. (2014). Effect of moisture on breakdown voltage and structure of palm based insulation oils. IEEE Trans. Dielect. Elect. Insul., 21(5), 2119-2126, https://doi.org/10.1109/TDEI.2014.004431.

11. Abdelmalik, A. A. (2014). Chemically modified palm kernel oil ester: A possible sustainable alternative insulating fluid. Sustain. Mater. Technol., 1, 42-51, https://doi.org/10.1016/j.susmat.2014.06.001.

12. Garba, Z. N., Gimba, C. E. \& Emmanuel, P. (2013). Production and characterisation of biobased transformer oil from Jatropha curcas seed. J. Phys. Sci., 24(2), 49-61.

13. Ilyas, S. M. (2006). Study on the characteristics of Jatropha and Ricinnus seed oils as liquid insulating materials. Ann. Rep. Conf. Elect. Insul. Dielectr. Phen., 162-165, https://doi.org/10.1109/CEIDP.2006.312086.

14. Abdelmalik, A. A. et al. (2011). Synthesis of a base-stock for electrical insulating fluid based on palm kernel oil. Ind. Crops Prod., 33, 532-536, https://doi.org/10.1016/j.indcrop.2010.11.019.

15. Dijkstra, A. J. \& Opstal, M. V. (1987). The total degumming process. J. Am. Oil Chem. Soc., 66, 1002-1009, https://doi.org/10.1007/BF02682627.

16. ASTM. (2005). Standard test method for pour point of petroleum products. New York: ASTM.

17. Abdelmalik, A. A., Fothergill, J. C. \& Dodd, S. J. (2013). Aging of Kraft paper insulation in natural ester dielectric fluid. Paper presented at the Proceedings of the IEEE International Conference on Solid Dielectrics, 541-544, https://doi.org/10.1109/ICSD.2013.6619678.

18. Nynas. (2010). Transformer oil handbook. Stockholm: Nynas AB.

19. Young, H. D. \& Freedman, R. A. (1996). University physics, 9th ed. London: Addison-Wesley Publishing Company.

20. Bartnikas, R. (1999). Permittivity and loss of insulating liquid. In Bartinikas, R. (Ed.). Engineering dielectrics, vol. 3. London: ASTM, 3-146.

21. McShane, C. P. et al. (2002). Aging of Kraft paper in natural ester dielectric fluid. Paper presented at the Proceedings of the IEEE International Conference on Dielectric Liquids, 173-177, https://doi.org/10.1109/ ICDL.2002.1022722.

22. Oommen, T. V. \& Prevost, T. A. (2011). Cellulose insulation in oil-filled power transformers: Maintaining insulation integrity and life. IEEE Elect. Insul. Mag., 22, 5-14, https://doi.org/10.1109/MEI.2006.1618996. 
23. Rapp, K. J., McShane, C. P. \& Luksich, J. (2005). Interaction mechanisms of natural ester dielectric fluid and Kraft paper. Paper presented at the Proceedings of the IEEE International Conference on Dielectric Liquids, https://doi.org/10.1109/ICDL.2005.1490108.

24. Frimpong, G. K., Oommen, T. V. \& Asano, R. (2011). A survey of aging characteristics of cellulose insulation in natural ester and mineral oil. IEEE Elect. Insul. Mag., 27, 36-48, https://doi.org/10.1109/MEI.2011.6025367.

25. Abdelmalik, A. A. (2015). Analysis of thermally aged insulation paper in a natural ester-based dielectric fluid. IEEE Trans. Dielect. Elect. Insul., 22(5), 2408-2414, https://doi.org/10.1109/TDEI.2014.004824.

26. Abdelmalik, A. A. et al. (2014). Charge transport in thermally aged paper impregnated with natural ester oil. IEEE Trans. Dielect. Elect. Insul., 21(5), 2318-2328, https://doi.org/10.1109/TDEI.2014.004316.

27. Dissado, L. A. \& Hill, R. M. (1983). A cluster approach to the structure of imperfect materials and their relaxation spectroscopy. Proc. R. Soc. Lond. A, 390, 131-180, https://doi.org/10.1098/rspa.1983.0125.

28. Stuart, B. (2004). Infrared spectroscopy: Fundamentals and application. Washington: John Willey \& Sons, https://doi.org/10.1002/0470011149. 